\title{
LIFE CYCLE ASSESSMENT OF INTEGRATED EXPLOITATION TECHNOLOGY FOR TAILINGS IN BAYAN OBO MINE, CHINA
}

\author{
WANG, L. ${ }^{1}-$ JIAO, G. H. ${ }^{1}-$ LU, H. S. $.^{1,2^{*}}-$ WANG, Q. Q. ${ }^{3 *}$ \\ ${ }^{I}$ Key Laboratory of Integrated Exploitation of Bayan Obo Multi-Metal Resources, Inner \\ Mongolia University of Science and Technology, Baotou 014010, China \\ ${ }^{2}$ School of Economics and Management, Inner Mongolia University of Science and Technology, \\ Baotou 014010, China \\ ${ }^{3}$ Institute of Mining, Inner Mongolia University of Science and Technology, \\ Baotou 014010, China \\ *Corresponding authors \\ e-mail:wanglu85@163.com \\ (Received $12^{\text {th }}$ Feb 2019; accepted $6^{\text {th }}$ Mar 2019)
}

\begin{abstract}
Bayan Obo mine in China is the largest iron-niobium-rare-earth symbiotic and associated mine in the world. Its exploitation has brought great economic benefit over the years. At the same time, many environmental problems arise. One of the most serious problems is tailings. Based on the theory and method of life cycle assessment, the environmental problems caused by integrated exploitation technology for tailings in Bayan Obo mine have been studied. Environmental impact assessment method TRACI 2.1 developed by Environmental Protection Agency of the United States has been chosen in this study. Through the study of resource consumption, energy consumption, pollution input by raw materials, pollution from industrial emissions etc. of each process, 10 types of environmental impacts have been studied and their values have been calculated. Results show that the main environmental impact types are Human toxicity non-cancer, Human toxicity cancer, Ecotoxicity, Global warming air, Human health particulate air, respectively. And the pollution input by raw materials is much greater than that from industrial emission, which provides a typical case and support for green purchasing, green manufacturing, process improving etc.
\end{abstract}

Keywords: life cycle impact assessment; life cycle inventory; environmental load; rare-earth; green manufacturing

\section{Introduction}

Bayan Obo mine, the largest iron-niobium-rare-earth symbiotic and associated deposit in the world, is located $149 \mathrm{~km}$ north of Baotou city, Inner Mongolia Autonomous region, China (Fig. 1). Beside rare-earth resources, the mine also contains other elements of interest, including iron, niobium, scandium, fluorite, sulphur and potassium etc. These resources also have an extremely high integrated exploitation value (Smith et al., 2015). Bayan Obo tailings ponds accumulated a large amount of iron tailings and rare-earth tailings due to the large scale mining for half a century. The integrated exploitation technology is a comprehensive method including beneficiation method and metallurgical method, which can extract the remaining useful elements from the tailings of Bayan Obo mine.

Six million tons oxidized ores are mined by Bayan Obo mine each year and 3.8618 million tons tailings are produced (Li et al., 2015a). These tailings bring a series of environmental problems including land occupation, radiation, dust pollution and groundwater pollution etc. The remaining elements in tailings such as iron, rare-earth, sulphur, fluorine, niobium, scandium etc. still have an extremely high recoverable 
value. Recycling these elements not only can generate considerable economic benefits, but also can greatly reduce environmental hazards. Currently, environmental problems caused by tailings generally are used some methods called "End-of-pipe Treatment". These methods cure the symptoms, not the disease-temporary medical relief. However, using the Life Cycle Assessment Method to analyze environmental problems produced in the process of integrated exploitation of tailings, can calculate their environmental pollution values in advance from the beginning of technology designing and achieve the goal of green production from the origin.

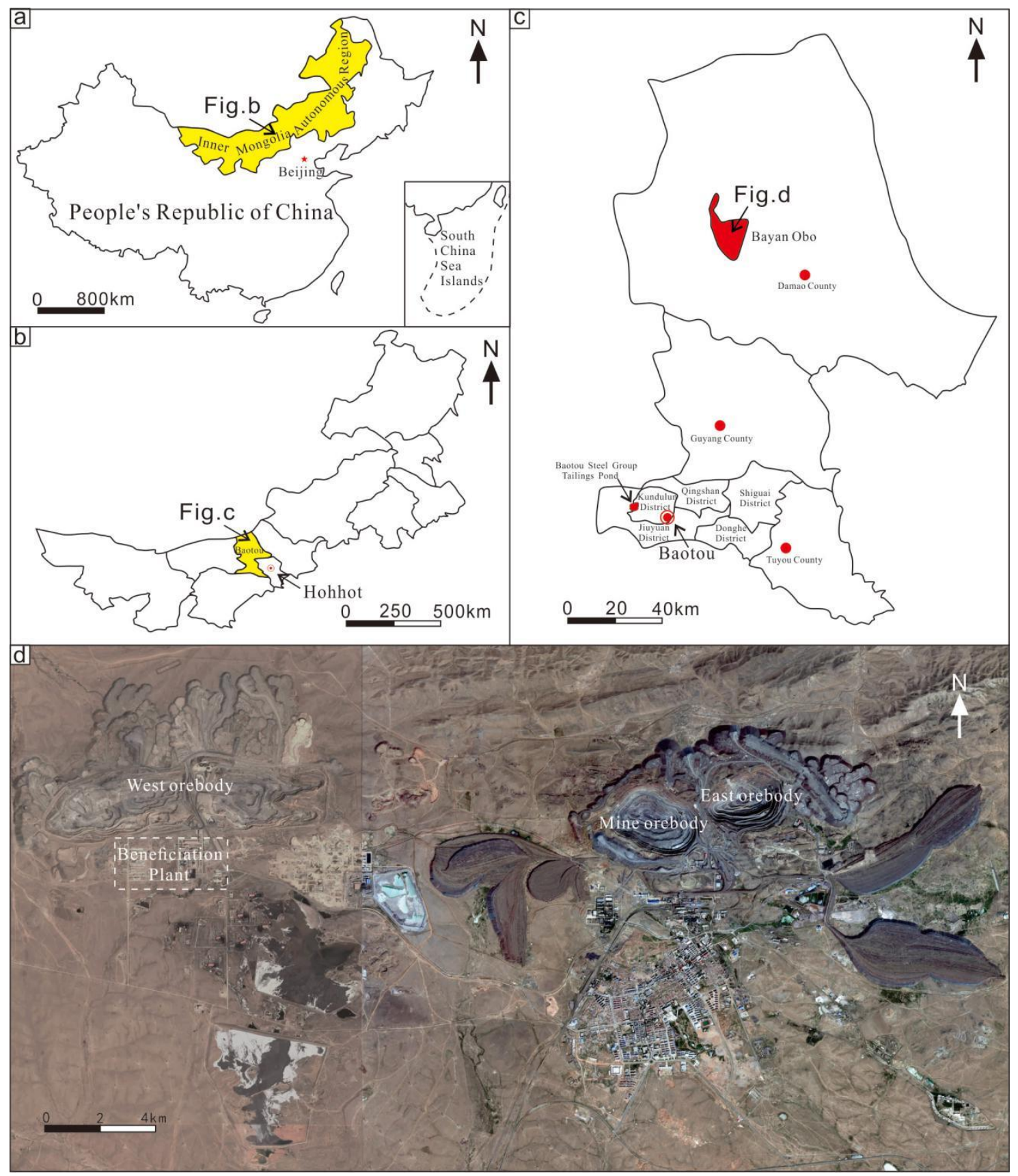

Figure 1. Location map of Bayan Obo mine. a Location of Inner Mongolia Autonomous Region. $\boldsymbol{b}$ Location of Baotou City. $\boldsymbol{c}$ Location of Bayan Obo mine.d Satellite image map of Bayan Obo mine (Base map from Google Earth) 
There are a lot of assessment methods at present (Ni et al., 2018; Zhai et al., 2018; Zhao et al., 2018; Zhou et al., 2018; Li et al., 2017; Yang et al., 2017; Jin et al., 2015; Li et al., 2015b; Song et al., 2015; Yan et al., 2014). Most of the methods are based on the current situation, and only the Life Cycle Assessment (LCA) starts from the source. LCA method assesses the entire life cycle of a product, technique, or activity in relation to its environmental impact, including the initial collection of the raw material, the production, transportation, sale, use, recycling, maintenance, and disposal of the product. The life cycle assessment method is an advanced method used to determine the comprehensive environmental impact of a process. It has been widely applied as an advanced method in modern environmental management (Mazzi et al., 2017).

At present, life cycle assessment of rare-earth is mainly on rare-earth oxide, neodymium-iron-boron $(\mathrm{NdFeB})$ permanent magnet material and related products containing rare-earth elements at home and abroad (Vahidi and Zhao, 2017, 2018; Zapp et al., 2018; Lima et al., 2018; Weng and Mudd, 2017; Schulze et al., 2017; Vahidi et al., 2016; Weng et al., 2016; Jin et al., 2016; Zaimes et al., 2015; Li et al., 2015c; Koltun and Tharumarajah, 2014; Navarro and Zhao, 2014; Sprecher et al., 2014). However life cycle assessment for tailings integrated exploitation of Bayan Obo mine is rare. This study can quantitatively analyze the environmental influence of useful elements in tailings in the process of integrated exploitation, which is benefit for improving mineral processing technology and achieve green purchasing (Liobikienè et al., 2017) and green manufacturing (Ahn et al, 2013). Meanwhile, it provides the basis for improvement and optimization of integrated exploitation technology of Bayan Obo mine.

\section{Study methods}

\section{Assessment method}

At present, the LCA methods mainly include CML (Centrum voor Milieukunde Leiden), EDIP (Environmental Design of Industrial Products), EI (Eco-Indicator), EPS (Environmental Priority Strategy), ES (Eco-Scarcity), ReCiPe, BEES (Building for Environment and Economic Sustainability), NOGEPA (Netherlands Oil and Gas Exploration and Production Association), TRACI (Tool for the Reduction and Assessment of Chemical and other Environmental) and so on. In CML method, weight of environmental impact factor mainly is the global average value, which will bring some error if it is used in a specific area. EDIP method is similar to CML method, which will has some error if a specific country is studied. Meanwhile, due to the early development, this method lacks timely update. EI and EPS methods have few environmental impact types, which cannot cover overall environmental impact. Using ES method requires some adjustments according to the change of country or area. ReCiPe method is not perfect in some calculation methods. BEES and NOGEPA are two methods that are developed on TRACI. The improved parts of these methods are limited in use and not widely applicable.

Based on the reasons mentioned above, this study selected TRACI 2.1 assessment method developed by Environmental Protection Agency of the United States (USEPA) in 2002 to assess the environmental influence of tailings integrated exploitation technology for Bayan Obo mine in China. This assessment method was developed for sustainable indexes, LCA, industrial ecology and impact assessment of process design, which was a kind of impact assessment methods for treating chemical emissions, 
resource utilization and environmental load source etc. Environmental impact factors considered by TRACI 2.1 were 10 types: AD (Acidification), EP (Eutrophication), GWA (Global Warming Air), ODA (Ozone Depletion Air), HHPA (Human Health Particulate Air), HTC (Human toxicity cancer), HTNC (Human toxicity non-cancer), ET (Ecotoxicity), SA (Smog Air) and RFF (Resources, Fossil fuels). The characterization unit, normalization references and weight factors of TRACI 2.1 are seen in Table 1.

Table 1. Classification, normalization references and weight factors of TRACI 2.1

\begin{tabular}{|c|c|c|c|}
\hline $\begin{array}{l}\text { Environmental } \\
\text { impact type }\end{array}$ & Characterization unit & $\begin{array}{c}\text { Normalization } \\
\text { reference } /\left(\mathrm{kg} \cdot \text { person }^{-1} \cdot \mathbf{a}^{-1}\right)\end{array}$ & $\begin{array}{l}\text { Weight } \\
\text { factor }\end{array}$ \\
\hline ET & CTUe & $1.1 \mathrm{E}+04$ & 6.7 \\
\hline HTC & CTUh & $4.8 \mathrm{E}-05$ & 7.8 \\
\hline HTNC & CTUh & $1.0 \mathrm{E}-03$ & 7.8 \\
\hline RFF & MJ surplus energy & $1.9 \mathrm{E}+04$ & 6.2 \\
\hline HHPA & kg PM2.5-Equiv & $3.0 \mathrm{E}+01$ & 6.6 \\
\hline ODA & kg CFC 11-Equiv & $1.5 \mathrm{E}-01$ & 5.2 \\
\hline SA & $\mathrm{kg} \mathrm{O}_{3}$-Equiv & $1.5 \mathrm{E}+03$ & 6.9 \\
\hline GWA & $\mathrm{kg} \mathrm{CO}_{2}$-Equiv & $2.4 \mathrm{E}+04$ & 8.9 \\
\hline $\mathrm{AD}$ & $\mathrm{kg} \mathrm{SO}_{2}$-Equiv & $9.5 \mathrm{E}+01$ & 5.8 \\
\hline EP & kg N-Equiv & $2.0 \mathrm{E}+01$ & 6.6 \\
\hline
\end{tabular}

Data from USEPA (Norris, 2002) https://nepis.epa.gov/Adobe/PDF/P100HN53.pdf

\section{Data quality}

The data related to integrated exploitation technology of Bayan Obo tailings were mainly acquired from patents (Zhang et al., 2015), statistical yearbooks (Baotou steel group, 2016; Baotou steel group, 2014), published books and literatures (Yang, 2017; Lu and Liu, 2016; Hu, 2014; Ji, 2013; Zhang et al., 2010; Xuan et al., 2009; Cheng et al., 2007; Che and Yu, 2006; Mei et al., 2006; Zhang et al., 2002; Xie et al., 2000; Tian and $\mathrm{Lu}, 1999$ ), and real production data of enterprises. The data acquisition time is from 2014-2018 (The application of integrated exploitation technology to Bayan Obo tailings starts from 2014). The data also had been logged by Baotou Iron \& Steel (Group) Co. Ltd.

The data can be divided into four types: energy, material, emission and front-end data. The first two data had been acquired from beneficiation plant of Bayan Obo mine, whose GPS coordinates is $41^{\circ} 47^{\prime} 30^{\prime \prime} \mathrm{N}$ and $109^{\circ} 51^{\prime} 51^{\prime \prime} \mathrm{E}$. The data of pollutant emission were acquired from environmental impact assessment report of integrated exploitation project for Bayan Obo tailings in 2016 and the People's Republic of China National Standard "Emission Standards of Pollutants from Rare Earths Industry" (Liu et al., 2015; Hu et al., 2013; Chen et al., 2012; Xiao et al., 2003).

Front-end data used in the study were from $\mathrm{GaBi}$ database. $\mathrm{GaBi}$ covers more than 12000 life cycle inventory data, which is the largest life cycle inventory data set, covered more than 40 industries in the world so far (the database has been updated to 2018). Data of electricity, steam and water were from the China mean value; input of other materials were from global mean value, European Union (EU) mean value, or Germany mean value. Because of the similar energy structure between China and 
Germany whose energy supplies mostly have been dominated by coal (Bonatz et al., 2019), the environmental impact of material production in Germany can substitute that of China. If $\mathrm{GaBi}$ database lacks China mean value, global mean value and EU mean value, Germany data were chosen, which can approximately substitute China data (Mo and Zhang, 2003).

For materials not in the database, we created some new data models or used some similar materials in database to substitute the original materials (Cao, 2017).

\section{Goal and scope}

Based on the integrated exploitation technology of tailings in Bayan Obo mine as the research goal, according to the collected data and materials, this study conducts a study on LCA of this technology. The purpose was to analyze environmental load, environmental impact types in each production stage and identify the key processes causing pollution and environmental impact types, which can provide basic data for life cycle assessment of integrated exploitation technology of tailings in Bayan Obo mine. Meanwhile, it can promote green purchasing, green manufacturing and sustainable development of rare-earth industry and tailings integrated exploitation industry.

In this study of LCA, system functional unit has been chosen as 3.8618 million tons of rare-earth tailings, which is an average quantity of tailings annual output. System boundary was from rare-earth tailings to scandium concentrate (500 ppm) (Fig. 2), which included processes of rare-earth flotation, iron flotation, sulfur flotation, fluorite flotation, niobium flotation and scandium magnetic separation etc. namely comprehensively recycling rare-earth concentrate, iron concentrate, sulfur concentrate, fluorite concentrate, niobium concentrate and scandium concentrate from rare-earth tailings.

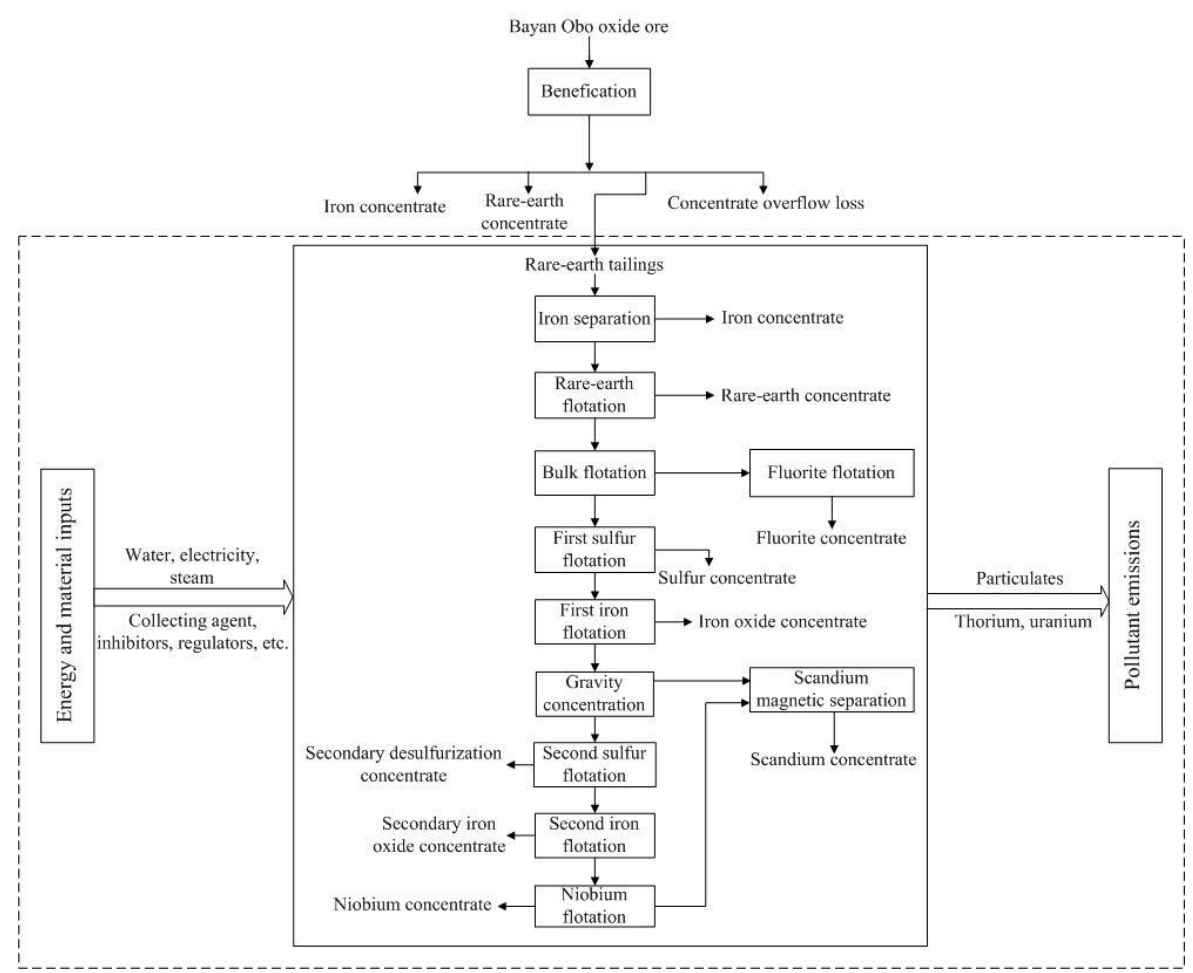

Figure 2. LCA system boundary of tailings integrated exploitation technology in Bayan Obo mine 


\section{Life cycle inventory (LCI)}

Integrated exploitation technology of tailings in Bayan Obo mine is a kind of process which can comprehensively recycle rare-earth concentrate, iron concentrate, sulfur concentrate, fluorite concentrate, niobium concentrate and scandium concentrate from Bayan Obo mine. In this technology, wastewater had achieved zero discharge. Types of energy included electricity, water and steam. Raw materials consumption mainly included collecting agents, inhibitors, regulators etc. in each beneficiation process. Pollutant discharge mainly included thorium, uranium, sulfur dioxide, carbon dioxide etc. The LCI data is shown in Figure 3.

\section{Life cycle impact assessment (LCIA)}

LCIA was the key procedure of LCA. This stage needs to quantitatively assess potential environmental impacts. At present, the methods used internationally are mainly qualitative analysis and quantitative calculation, which generally include three stages: characterization, normalization and weighting.

\section{Characterization}

Characterization was quantitatively analysis contributions of each environmental factor on specific environmental impact types, and then converted them into unified unit. Characterization results are shown in Table 2.

Table 2. Characterization results of LCI data

\begin{tabular}{|c|c|c|c|c|c|c|}
\hline Types & Iron separation & $\begin{array}{c}\text { Rare-earth } \\
\text { flotation }\end{array}$ & $\begin{array}{c}\text { Bulk } \\
\text { flotation }\end{array}$ & $\begin{array}{l}\text { Fluorite } \\
\text { flotation }\end{array}$ & $\begin{array}{c}\text { First sulfur } \\
\text { flotation }\end{array}$ & $\begin{array}{c}\text { First iron } \\
\text { flotation }\end{array}$ \\
\hline $\mathrm{AD} / \mathrm{kg}$ & $3.48 \mathrm{E}+05$ & $5.98 \mathrm{E}+05$ & $9.70 \mathrm{E}+05$ & $1.07 \mathrm{E}+06$ & $2.05 \mathrm{E}+04$ & $1.55 \mathrm{E}+06$ \\
\hline ET/CTUe & $3.41 \mathrm{E}+08$ & $4.06 \mathrm{E}+08$ & $1.56 \mathrm{E}+08$ & $1.19 \mathrm{E}+08$ & $1.41 \mathrm{E}+07$ & $3.39 \mathrm{E}+08$ \\
\hline $\mathrm{EP} / \mathrm{kg}$ & $1.34 \mathrm{E}+05$ & $1.44 \mathrm{E}+05$ & $7.45 \mathrm{E}+04$ & $1.17 \mathrm{E}+05$ & $1.17 \mathrm{E}+04$ & $1.30 \mathrm{E}+05$ \\
\hline $\mathrm{GWA} / \mathrm{kg}$ & $9.24 \mathrm{E}+07$ & $1.46 \mathrm{E}+08$ & $2.40 \mathrm{E}+08$ & $2.53 \mathrm{E}+08$ & $7.92 \mathrm{E}+06$ & $3.32 \mathrm{E}+08$ \\
\hline HHPA $/ \mathrm{kg}$ & $1.42 \mathrm{E}+05$ & $1.91 \mathrm{E}+05$ & $3.18 \mathrm{E}+05$ & $3.54 \mathrm{E}+05$ & $1.83 \mathrm{E}+04$ & $4.60 \mathrm{E}+05$ \\
\hline HTC/CTUh & $1.32 \mathrm{E}+00$ & $1.53 \mathrm{E}+00$ & $6.17 \mathrm{E}-01$ & $4.81 \mathrm{E}-01$ & $6.98 \mathrm{E}-02$ & $1.34 \mathrm{E}+00$ \\
\hline HTNC/CTUh & $1.90 \mathrm{E}+01$ & $2.91 \mathrm{E}+01$ & $3.37 \mathrm{E}+01$ & $3.56 \mathrm{E}+01$ & $8.17 \mathrm{E}-01$ & $5.03 \mathrm{E}+01$ \\
\hline ODA $/ \mathrm{kg}$ & $2.85 \mathrm{E}+00$ & $3.48 \mathrm{E}+00$ & $9.87 \mathrm{E}-01$ & $6.82 \mathrm{E}-01$ & $6.04 \mathrm{E}-02$ & $1.23 \mathrm{E}+00$ \\
\hline RFF/MJ & $7.53 \mathrm{E}+07$ & $8.40 \mathrm{E}+07$ & $5.98 \mathrm{E}+07$ & $6.18 \mathrm{E}+07$ & $5.51 \mathrm{E}+06$ & $6.34 \mathrm{E}+07$ \\
\hline $\mathrm{SA} / \mathrm{kg}$ & $4.10 \mathrm{E}+06$ & 7.07E+06 & $1.21 \mathrm{E}+07$ & $1.33 \mathrm{E}+07$ & $2.74 \mathrm{E}+05$ & $1.77 \mathrm{E}+07$ \\
\hline Types & $\begin{array}{c}\text { Gravity } \\
\text { concentration }\end{array}$ & $\begin{array}{l}\text { Second sulfur } \\
\text { flotation }\end{array}$ & $\begin{array}{c}\text { Second iron } \\
\text { flotation }\end{array}$ & $\begin{array}{l}\text { Niobium } \\
\text { flotation }\end{array}$ & $\begin{array}{c}\text { Scandium } \\
\text { magnetic } \\
\text { separation }\end{array}$ & Total \\
\hline $\mathrm{AD} / \mathrm{kg}$ & $1.16 \mathrm{E}+04$ & $3.12 \mathrm{E}+03$ & $7.68 \mathrm{E}+05$ & $2.25 \mathrm{E}+05$ & $5.78 \mathrm{E}+03$ & $5.58 \mathrm{E}+06$ \\
\hline ET/CTUe & $8.59 \mathrm{E}+05$ & $3.43 \mathrm{E}+06$ & $1.32 \mathrm{E}+08$ & $8.24 \mathrm{E}+07$ & $2.97 \mathrm{E}+05$ & $1.59 \mathrm{E}+09$ \\
\hline $\mathrm{EP} / \mathrm{kg}$ & $4.16 \mathrm{E}+03$ & $2.50 \mathrm{E}+03$ & $5.44 \mathrm{E}+04$ & $3.43 E+04$ & $9.58 \mathrm{E}+02$ & $7.07 \mathrm{E}+05$ \\
\hline $\mathrm{GWA} / \mathrm{kg}$ & $6.29 \mathrm{E}+06$ & $1.11 \mathrm{E}+06$ & $1.72 \mathrm{E}+08$ & $4.58 \mathrm{E}+07$ & $2.10 \mathrm{E}+06$ & $1.30 \mathrm{E}+09$ \\
\hline HHPA $/ \mathrm{kg}$ & $2.43 \mathrm{E}+04$ & $1.68 \mathrm{E}+03$ & $2.34 \mathrm{E}+05$ & $6.38 \mathrm{E}+04$ & $1.14 \mathrm{E}+04$ & $1.82 \mathrm{E}+06$ \\
\hline HTC/CTUh & $1.91 \mathrm{E}-02$ & $1.55 \mathrm{E}-02$ & $5.24 \mathrm{E}-01$ & $3.29 \mathrm{E}-01$ & 4.32E-03 & $6.25 \mathrm{E}+00$ \\
\hline HTNC/CTUh & 4.09E-01 & $1.42 \mathrm{E}-01$ & $2.49 \mathrm{E}+01$ & $7.19 \mathrm{E}+00$ & $1.89 \mathrm{E}-01$ & $2.01 \mathrm{E}+02$ \\
\hline $\mathrm{ODA} / \mathrm{kg}$ & $1.84 \mathrm{E}-05$ & $1.47 \mathrm{E}-02$ & 4.53E-01 & $3.27 \mathrm{E}-01$ & $3.87 \mathrm{E}-06$ & $1.01 \mathrm{E}+01$ \\
\hline RFF/MJ & $6.32 \mathrm{E}+06$ & $5.88 \mathrm{E}+05$ & $3.20 \mathrm{E}+07$ & $1.43 \mathrm{E}+07$ & $1.43 \mathrm{E}+06$ & $4.04 \mathrm{E}+08$ \\
\hline $\mathrm{SA} / \mathrm{kg}$ & $1.82 \mathrm{E}+05$ & $3.84 \mathrm{E}+04$ & $9.07 \mathrm{E}+06$ & $2.44 \mathrm{E}+06$ & $8.16 \mathrm{E}+04$ & $6.64 \mathrm{E}+07$ \\
\hline
\end{tabular}




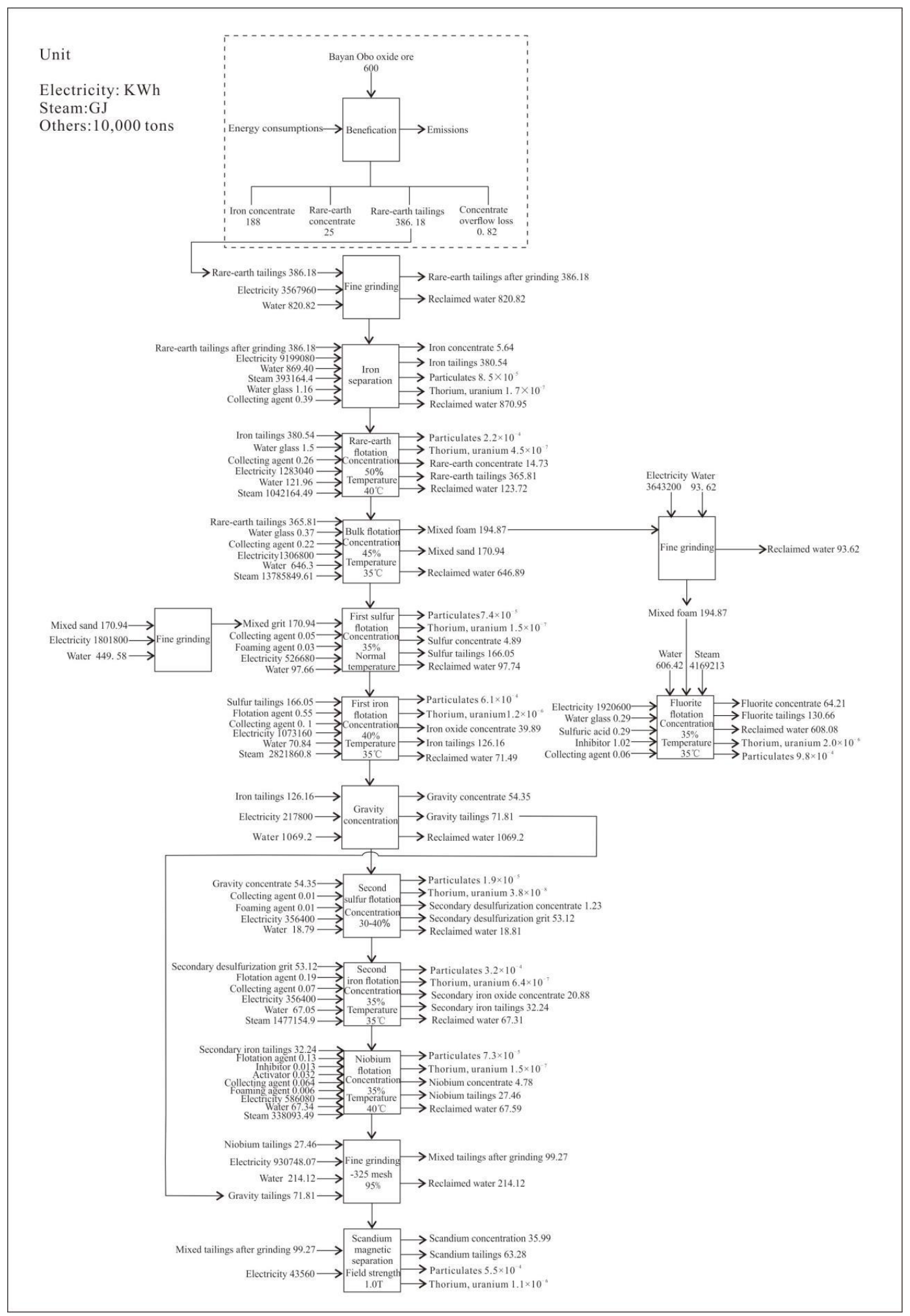

Figure 3. LCI data of integrated exploitation technology for tailings in Bayan Obo mine

\section{Normalization}

Normalization is a calculating process that using the acquired characterization values divided corresponding normalization reference values, respectively. In the end, this 
calculation can get some data without unit. After normalization process, these environmental impact types are comparable due to the removal of previous units. Normalization reference value of each environmental impact type is closely related to the severity of itself. Relative levels of various environmental impact types can be compared preliminarily after this procedure.

Normalization reference values of this study are provided by TRACI 2.1 in GaBi 8.0 software. The calculation results are shown in Table 3.

Table 3. Normalization results of LCI data

\begin{tabular}{|c|c|c|c|c|c|c|}
\hline Types & Iron separation & $\begin{array}{c}\text { Rare-earth } \\
\text { flotation }\end{array}$ & $\begin{array}{c}\text { Bulk } \\
\text { flotation }\end{array}$ & $\begin{array}{l}\text { Fluorite } \\
\text { flotation }\end{array}$ & $\begin{array}{c}\text { First sulfur } \\
\text { flotation }\end{array}$ & $\begin{array}{l}\text { First iron } \\
\text { flotation }\end{array}$ \\
\hline $\mathrm{AD}$ & $3.67 \mathrm{E}+03$ & $6.32 \mathrm{E}+03$ & $1.03 E+04$ & $1.13 \mathrm{E}+04$ & $2.17 \mathrm{E}+02$ & $1.64 \mathrm{E}+04$ \\
\hline ET & $3.11 \mathrm{E}+04$ & $3.70 \mathrm{E}+04$ & $1.42 \mathrm{E}+04$ & $1.08 \mathrm{E}+04$ & $1.29 \mathrm{E}+03$ & $3.09 \mathrm{E}+04$ \\
\hline EP & $6.48 \mathrm{E}+03$ & $6.96 \mathrm{E}+03$ & $3.61 \mathrm{E}+03$ & $5.70 \mathrm{E}+03$ & $5.64 \mathrm{E}+02$ & $6.28 \mathrm{E}+03$ \\
\hline GWA & $3.84 \mathrm{E}+03$ & $6.06 \mathrm{E}+03$ & $1.00 \mathrm{E}+04$ & $1.05 \mathrm{E}+04$ & $3.29 \mathrm{E}+02$ & $1.38 \mathrm{E}+04$ \\
\hline HHPA & $4.78 \mathrm{E}+03$ & $6.43 \mathrm{E}+03$ & $1.07 \mathrm{E}+04$ & $1.19 \mathrm{E}+04$ & $6.16 \mathrm{E}+02$ & $1.55 \mathrm{E}+04$ \\
\hline HTC & $2.66 \mathrm{E}+04$ & $3.08 \mathrm{E}+04$ & $1.25 \mathrm{E}+04$ & $9.80 \mathrm{E}+03$ & $1.41 \mathrm{E}+03$ & $2.71 \mathrm{E}+04$ \\
\hline HTNC & $1.84 \mathrm{E}+04$ & $2.81 \mathrm{E}+04$ & $3.27 \mathrm{E}+04$ & $3.46 \mathrm{E}+04$ & $7.93 \mathrm{E}+02$ & $4.88 \mathrm{E}+04$ \\
\hline ODA & $1.95 \mathrm{E}+01$ & $2.39 \mathrm{E}+01$ & $6.77 \mathrm{E}+00$ & $4.68 \mathrm{E}+00$ & 4.15E-01 & $8.42 \mathrm{E}+00$ \\
\hline RFF & $3.62 \mathrm{E}+03$ & $4.03 E+03$ & $2.99 \mathrm{E}+03$ & $3.12 \mathrm{E}+03$ & $2.76 \mathrm{E}+02$ & $3.20 \mathrm{E}+03$ \\
\hline SA & $2.83 \mathrm{E}+03$ & $4.88 \mathrm{E}+03$ & $8.36 \mathrm{E}+03$ & $9.19 \mathrm{E}+03$ & $1.89 \mathrm{E}+02$ & $1.23 \mathrm{E}+04$ \\
\hline Types & $\begin{array}{c}\text { Gravity } \\
\text { concentration }\end{array}$ & $\begin{array}{c}\text { Second } \\
\text { sulfur } \\
\text { flotation }\end{array}$ & $\begin{array}{c}\text { Second iron } \\
\text { flotation }\end{array}$ & $\begin{array}{l}\text { Niobium } \\
\text { flotation }\end{array}$ & $\begin{array}{c}\text { Scandium } \\
\text { magnetic } \\
\text { separation }\end{array}$ & Total value \\
\hline $\mathrm{AD}$ & $1.22 \mathrm{E}+02$ & $3.29 \mathrm{E}+01$ & $8.12 \mathrm{E}+03$ & $2.39 \mathrm{E}+03$ & $6.11 \mathrm{E}+01$ & $5.89 \mathrm{E}+04$ \\
\hline ET & $7.81 \mathrm{E}+01$ & $3.12 \mathrm{E}+02$ & $1.20 \mathrm{E}+04$ & $7.51 \mathrm{E}+03$ & $2.70 \mathrm{E}+01$ & $1.45 \mathrm{E}+05$ \\
\hline EP & $2.04 \mathrm{E}+02$ & $1.21 \mathrm{E}+02$ & $2.64 \mathrm{E}+03$ & $1.66 \mathrm{E}+03$ & $4.69 \mathrm{E}+01$ & $3.43 \mathrm{E}+04$ \\
\hline GWA & $2.62 \mathrm{E}+02$ & $4.62 \mathrm{E}+01$ & $7.17 \mathrm{E}+03$ & $1.90 \mathrm{E}+03$ & $8.74 \mathrm{E}+01$ & $5.40 \mathrm{E}+04$ \\
\hline HHPA & $8.20 \mathrm{E}+02$ & $5.66 \mathrm{E}+01$ & $7.91 \mathrm{E}+03$ & $2.16 \mathrm{E}+03$ & $3.86 \mathrm{E}+02$ & $6.13 \mathrm{E}+04$ \\
\hline HTC & $3.97 \mathrm{E}+02$ & $3.11 \mathrm{E}+02$ & $1.06 \mathrm{E}+04$ & $6.64 \mathrm{E}+03$ & $9.01 \mathrm{E}+01$ & $1.26 \mathrm{E}+05$ \\
\hline HTNC & $3.97 \mathrm{E}+02$ & $1.38 \mathrm{E}+02$ & $2.42 \mathrm{E}+04$ & $6.97 \mathrm{E}+03$ & $1.84 \mathrm{E}+02$ & $1.95 \mathrm{E}+05$ \\
\hline ODA & $1.26 \mathrm{E}-04$ & $1.01 \mathrm{E}-01$ & $3.11 \mathrm{E}+00$ & $2.24 \mathrm{E}+00$ & $2.65 \mathrm{E}-05$ & $6.92 \mathrm{E}+01$ \\
\hline RFF & $3.26 \mathrm{E}+02$ & $2.85 \mathrm{E}+01$ & $1.62 \mathrm{E}+03$ & $7.16 \mathrm{E}+02$ & $7.36 \mathrm{E}+01$ & $2.00 \mathrm{E}+04$ \\
\hline SA & $1.26 \mathrm{E}+02$ & $2.65 \mathrm{E}+01$ & $6.25 \mathrm{E}+03$ & $1.68 \mathrm{E}+03$ & $5.63 \mathrm{E}+01$ & $4.59 \mathrm{E}+04$ \\
\hline
\end{tabular}

\section{Weighting}

Through characterization and normalization, summary value of specific assessment subject on each environmental impact type can be gotten. Each environmental impact type is assigned specific weighting factor, on the basis, normalization values were weighted. Then the comprehensive impact values get. Through this procedure, the total environmental impact of study object can be assessed. Meanwhile, the proportions of different environmental impact types in the overall environmental impact can be obtained. Through this result, the impact degree of different environmental impact types to the total environment can be analyzed and quantitative data can be calculated.

TRACI 2.1 environmental impact weight-values were used to get quantitative results of the total environmental impact. Calculation results are shown in Table 4. 
Table 4. Weighting results of each environmental impact type

\begin{tabular}{|c|c|c|c|c|c|c|}
\hline Types & Iron separation & $\begin{array}{c}\text { Rare-earth } \\
\text { flotation }\end{array}$ & $\begin{array}{c}\text { Bulk } \\
\text { flotation }\end{array}$ & $\begin{array}{l}\text { Fluorite } \\
\text { flotation }\end{array}$ & \begin{tabular}{|c|}
$\begin{array}{c}\text { First sulfur } \\
\text { flotation }\end{array}$ \\
\end{tabular} & $\begin{array}{c}\text { First iron } \\
\text { flotation }\end{array}$ \\
\hline $\mathrm{AD}$ & $2.14 \mathrm{E}+04$ & $3.67 \mathrm{E}+04$ & $5.95 \mathrm{E}+04$ & $6.56 \mathrm{E}+04$ & $1.25 \mathrm{E}+03$ & $8.26 \mathrm{E}+04$ \\
\hline ET & $2.08 \mathrm{E}+05$ & $2.48 \mathrm{E}+05$ & $9.54 \mathrm{E}+04$ & $7.26 \mathrm{E}+04$ & $8.63 \mathrm{E}+03$ & $6.46 \mathrm{E}+04$ \\
\hline EP & $4.28 \mathrm{E}+04$ & $4.59 \mathrm{E}+04$ & $2.38 \mathrm{E}+04$ & $3.75 \mathrm{E}+04$ & $3.72 \mathrm{E}+03$ & $1.82 \mathrm{E}+04$ \\
\hline GWA & $3.42 \mathrm{E}+04$ & $5.39 \mathrm{E}+04$ & $8.89 \mathrm{E}+04$ & $9.36 \mathrm{E}+04$ & $2.93 \mathrm{E}+03$ & $1.19 \mathrm{E}+05$ \\
\hline HHPA & $3.15 \mathrm{E}+04$ & $4.24 \mathrm{E}+04$ & $7.09 \mathrm{E}+04$ & $7.88 \mathrm{E}+04$ & $4.06 \mathrm{E}+03$ & $9.47 \mathrm{E}+04$ \\
\hline HTC & $2.08 \mathrm{E}+05$ & $2.41 \mathrm{E}+05$ & $9.76 \mathrm{E}+04$ & $7.65 \mathrm{E}+04$ & $1.10 \mathrm{E}+04$ & $6.41 \mathrm{E}+04$ \\
\hline HTNC & $1.43 E+05$ & $2.19 \mathrm{E}+05$ & $2.55 \mathrm{E}+05$ & $2.70 \mathrm{E}+05$ & $6.18 \mathrm{E}+03$ & $3.25 \mathrm{E}+05$ \\
\hline ODA & $1.02 \mathrm{E}+02$ & $1.24 \mathrm{E}+02$ & $3.52 \mathrm{E}+01$ & $2.43 \mathrm{E}+01$ & $2.16 \mathrm{E}+00$ & $1.08 \mathrm{E}+01$ \\
\hline RFF & $2.25 \mathrm{E}+04$ & $2.50 \mathrm{E}+04$ & $1.85 \mathrm{E}+04$ & $1.93 \mathrm{E}+04$ & $1.71 \mathrm{E}+03$ & $1.72 \mathrm{E}+04$ \\
\hline SA & $1.95 \mathrm{E}+04$ & $3.37 \mathrm{E}+04$ & $5.77 \mathrm{E}+04$ & $6.33 \mathrm{E}+04$ & $1.31 \mathrm{E}+03$ & $7.91 \mathrm{E}+04$ \\
\hline Types & $\begin{array}{c}\text { Gravity } \\
\text { concentration }\end{array}$ & $\begin{array}{l}\text { Second } \\
\text { sulfur } \\
\text { flotation }\end{array}$ & $\begin{array}{c}\text { Second iron } \\
\text { flotation }\end{array}$ & $\begin{array}{l}\text { Niobium } \\
\text { flotation }\end{array}$ & $\begin{array}{l}\text { Scandium } \\
\text { magnetic } \\
\text { separation }\end{array}$ & Total value \\
\hline $\mathrm{AD}$ & $7.10 \mathrm{E}+02$ & $1.91 \mathrm{E}+02$ & $4.28 \mathrm{E}+04$ & $1.09 \mathrm{E}+04$ & $3.54 \mathrm{E}+02$ & $3.22 \mathrm{E}+05$ \\
\hline ET & $5.23 E+02$ & $2.09 \mathrm{E}+03$ & $3.12 \mathrm{E}+04$ & $1.67 \mathrm{E}+04$ & $1.81 \mathrm{E}+02$ & $7.48 \mathrm{E}+05$ \\
\hline EP & $1.35 \mathrm{E}+03$ & $7.97 \mathrm{E}+02$ & $9.36 \mathrm{E}+03$ & $5.44 \mathrm{E}+03$ & $3.10 \mathrm{E}+02$ & $1.89 \mathrm{E}+05$ \\
\hline GWA & $2.33 \mathrm{E}+03$ & $4.12 \mathrm{E}+02$ & $6.23 \mathrm{E}+04$ & $1.59 \mathrm{E}+04$ & $7.78 \mathrm{E}+02$ & $4.74 \mathrm{E}+05$ \\
\hline HHPA & $5.41 \mathrm{E}+03$ & $3.73 \mathrm{E}+02$ & $4.95 \mathrm{E}+04$ & $1.24 \mathrm{E}+04$ & $2.54 \mathrm{E}+03$ & $3.93 \mathrm{E}+05$ \\
\hline HTC & $3.10 \mathrm{E}+03$ & $2.43 \mathrm{E}+03$ & $3.17 \mathrm{E}+04$ & $1.70 \mathrm{E}+04$ & $7.03 \mathrm{E}+02$ & $7.52 \mathrm{E}+05$ \\
\hline HTNC & $3.10 \mathrm{E}+03$ & $1.08 \mathrm{E}+03$ & $1.69 \mathrm{E}+05$ & $4.13 \mathrm{E}+04$ & $1.43 E+03$ & $1.43 \mathrm{E}+06$ \\
\hline ODA & $6.54 \mathrm{E}-04$ & 5.23E-01 & $4.76 \mathrm{E}+00$ & $3.87 \mathrm{E}+00$ & $1.38 \mathrm{E}-04$ & $3.08 \mathrm{E}+02$ \\
\hline RFF & $2.02 \mathrm{E}+03$ & $1.76 \mathrm{E}+02$ & $9.16 \mathrm{E}+03$ & $3.82 \mathrm{E}+03$ & $4.57 \mathrm{E}+02$ & $1.20 \mathrm{E}+05$ \\
\hline SA & $8.66 \mathrm{E}+02$ & $1.83 \mathrm{E}+02$ & $4.12 \mathrm{E}+04$ & $1.03 \mathrm{E}+04$ & $3.89 \mathrm{E}+02$ & $3.08 \mathrm{E}+05$ \\
\hline
\end{tabular}

\section{Results and interpretation}

According to the weighting results, environmental impact type values of integrated exploitation technology for tailings in Bayan Obo mine have been calculated (Fig. 4).

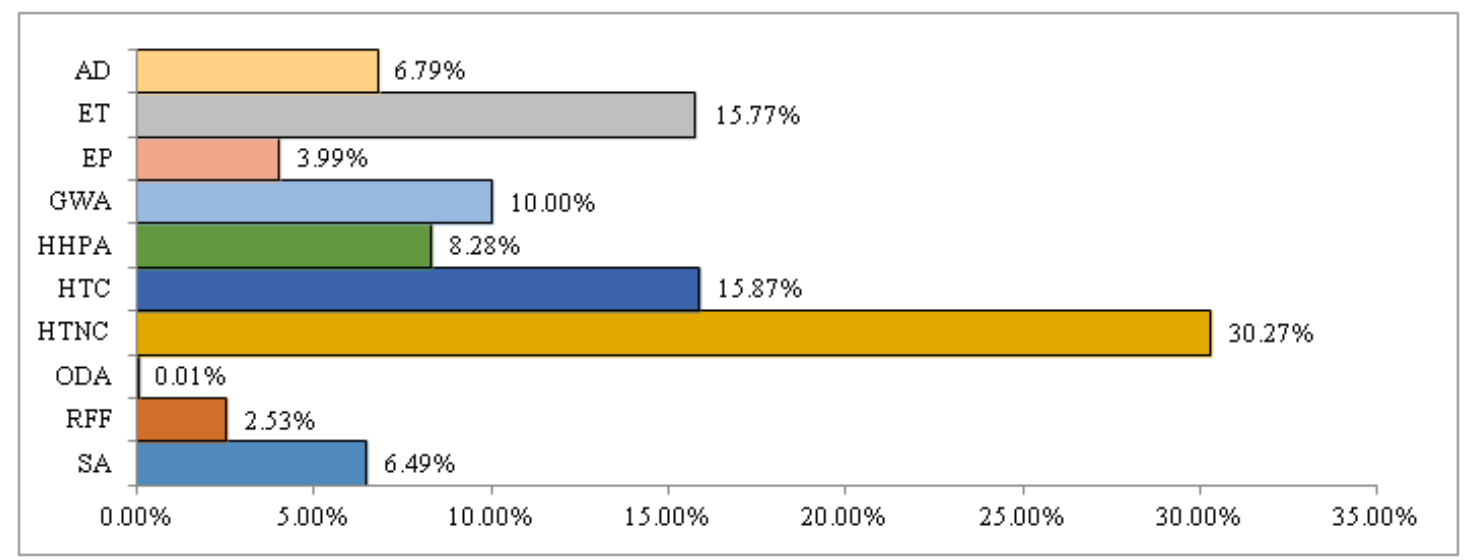

Figure 4. Proportion of each environmental impact type in the total environmental impact 
From Figure 4, the potential environmental impacts of integrated exploitation technology for tailings in Bayan Obo mine was HTNC, HTC, ET, GWA, HHPA, AD, SA, EP, RFF and ODA sorted by numerical value. Among these types, HTNC was the main environmental impact type, accounting for $30.27 \%$ of the total environmental impact. HTC was the next, accounting for $15.87 \%$ of the total environmental impact. ET, GWA and HHPA accounted for $15.77 \%, 10.00 \%$ and $8.28 \%$, respectively.

The most serious impact process in HTNC was iron flotation, whose contribution rate was $22.64 \%$ (Fig. 5). The contribution rate of fluorite flotation, bulk flotation and rare-earth flotation were $18.82 \%, 17.80 \%$ and $15.29 \%$, respectively. Steam was the main factor influencing HTNC in the stage of iron flotation, accounting for $96.33 \%$ (Fig. 6), which was mainly caused by organic matters discharge such as trichlopropane, trichloro ethylene etc.

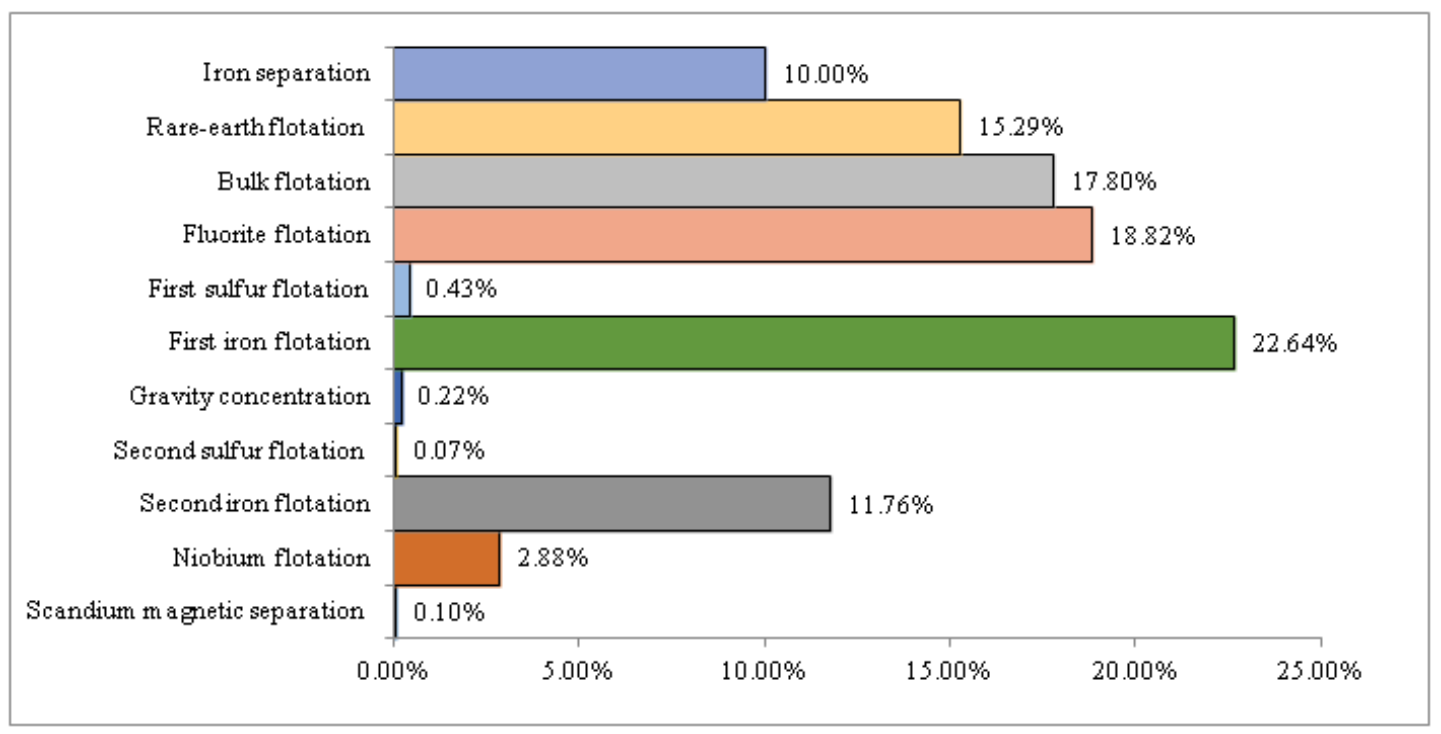

Figure 5. Proportion of HTNC in each process

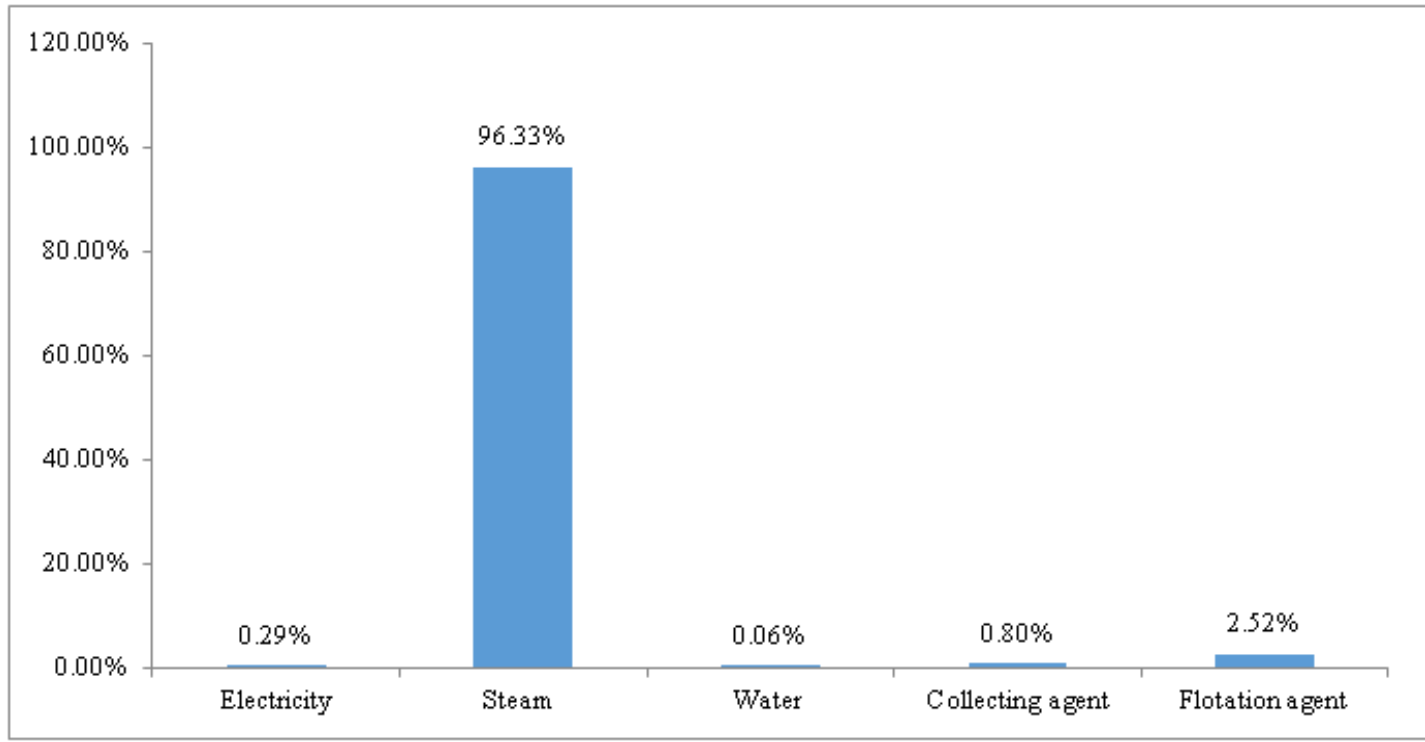

Figure 6. Proportion of environmental impact of each input in iron flotation in HTNC 
From the LCA results of integrated exploitation technology for tailings in Bayan Obo mine, the second environmental impact type is HTC, in which, the most serious impact process was rare-earth flotation (Fig. 7), accounting for $31.97 \%$ of the total environmental impact of HTC. The next was iron separation process, accounting for $27.62 \%$. Water glass was the main factor influencing HTC in the stage of rare-earth flotation, accounting for $83.16 \%$ (Fig. 8). The next was collecting agent, accounting for $11.87 \%$. The main reason was discharge of arsenite, cadmium and benzene etc. in the manufacturing process of water glass and collecting agent.

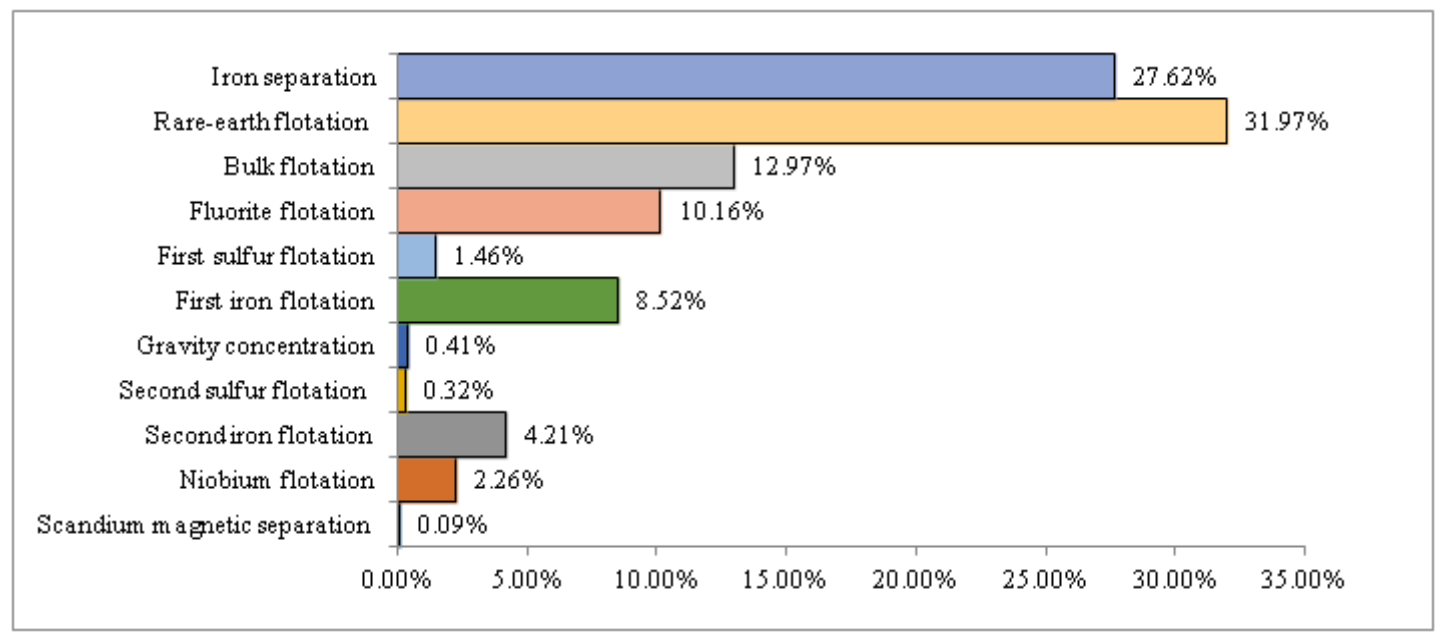

Figure 7. Proportion of HTC in each process

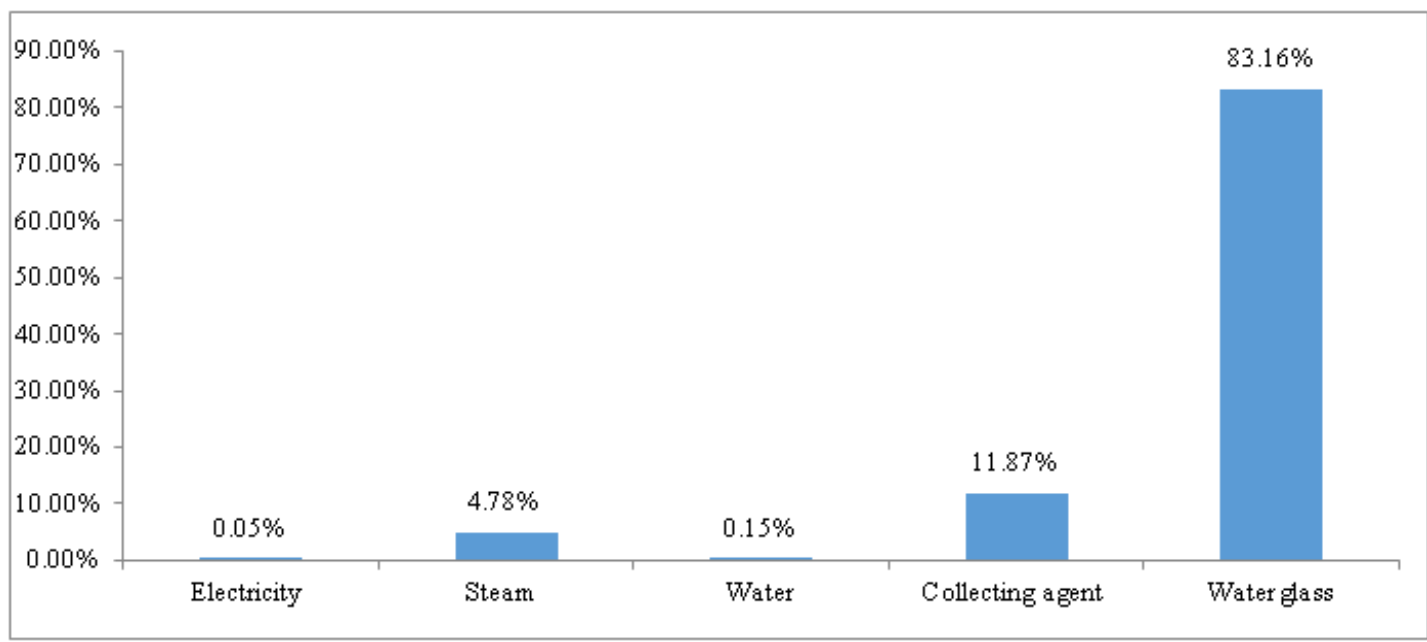

Figure 8. Proportion of environmental impact of each input in rare-earth flotation in HTC

The first environmental impact type, namely HTNC was mainly generated from iron flotation and the next was fluorite flotation. The second environmental impact type, namely HTC was mainly generated from rare-earth flotation and iron separation. The most serious impact in ET was rare-earth flotation and the next is iron separation. GWA was mainly generated from iron flotation and fluorite flotation. ODA was the least serious environmental impact among the total environmental impact types, which 
mainly generated from rare-earth flotation and iron separation (Fig. 9). From the above, the pollution caused by iron flotation was much higher than other processes.

The integrated exploitation technology for tailings in Bayan Obo mine covers 11 processes including iron separation, rare-earth flotation, bulk flotation, fluorite flotation, first sulfur flotation, first iron flotation, gravity concentration, second sulfur flotation, second iron flotation, niobium flotation, and scandium separation magnetic. The proportion of each environmental impact type in these processes is different (Fig. 10).

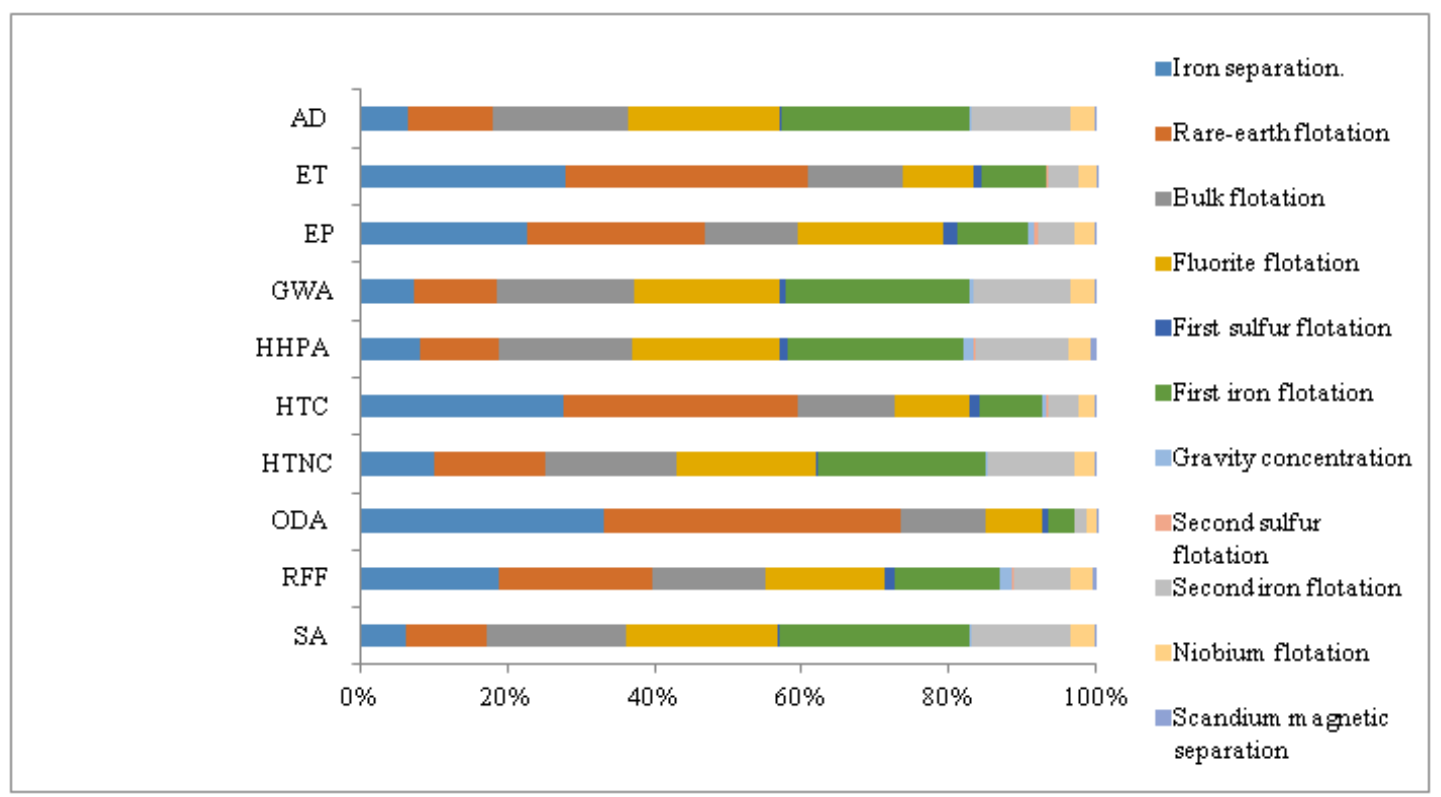

Figure 9. Proportion of environmental impact of each process in each environmental impact type

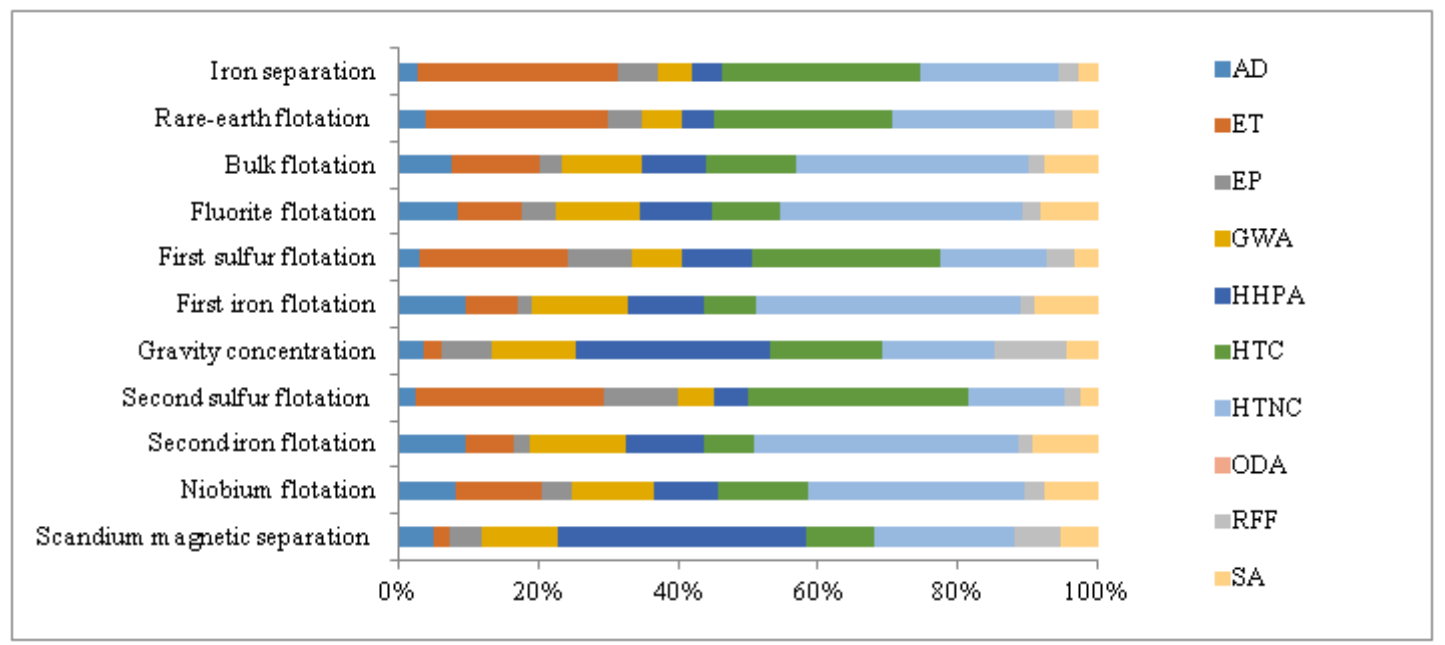

Figure 10. Proportion of each environmental impact type in each process

From the figure, in the processes of rare-earth flotation and iron separation, ET was the most important environmental impact type. HTC took second place. In the processes of bulk flotation and niobium flotation, HTNC was the major environmental impact 
type. HTC was the secondary. In the processes of first sulfur flotation and second sulfur flotation, HTC and ET were the major environmental impact types. In the processes of fluorite flotation, first iron flotation and second iron flotation, the most important environmental impact type was HTNC, and the next was GWA. However, in the process of gravity concentration, the main environmental impact type was HHPA, accounting for $27.88 \%$. The next was HTC, accounting for $15.97 \%$. In addition, in the process of scandium magnetic separation, HHPA and HTNC were the major environmental impact types, accounting for $35.59 \%$ and $20.06 \%$, respectively.

In LCA of integrated exploitation technology for tailings in Bayan Obo mine, proportion of each environmental impact type in the total environmental impact is different (Fig. 11). The environmental impact order of these processes was rare-earth flotation, iron flotation, fluorite flotation, bulk flotation and iron separation etc. Environmental impact of rare-earth flotation was the most serious, accounting for $19.94 \%$ in the total environmental impact. Iron flotation was the next, accounting for $18.23 \%$ in the total environmental impact. Scandium magnetic separation had the minimal impact on environment, accounting for $0.15 \%$. In the process of rare-earth flotation, the major environmental impact type was ET.

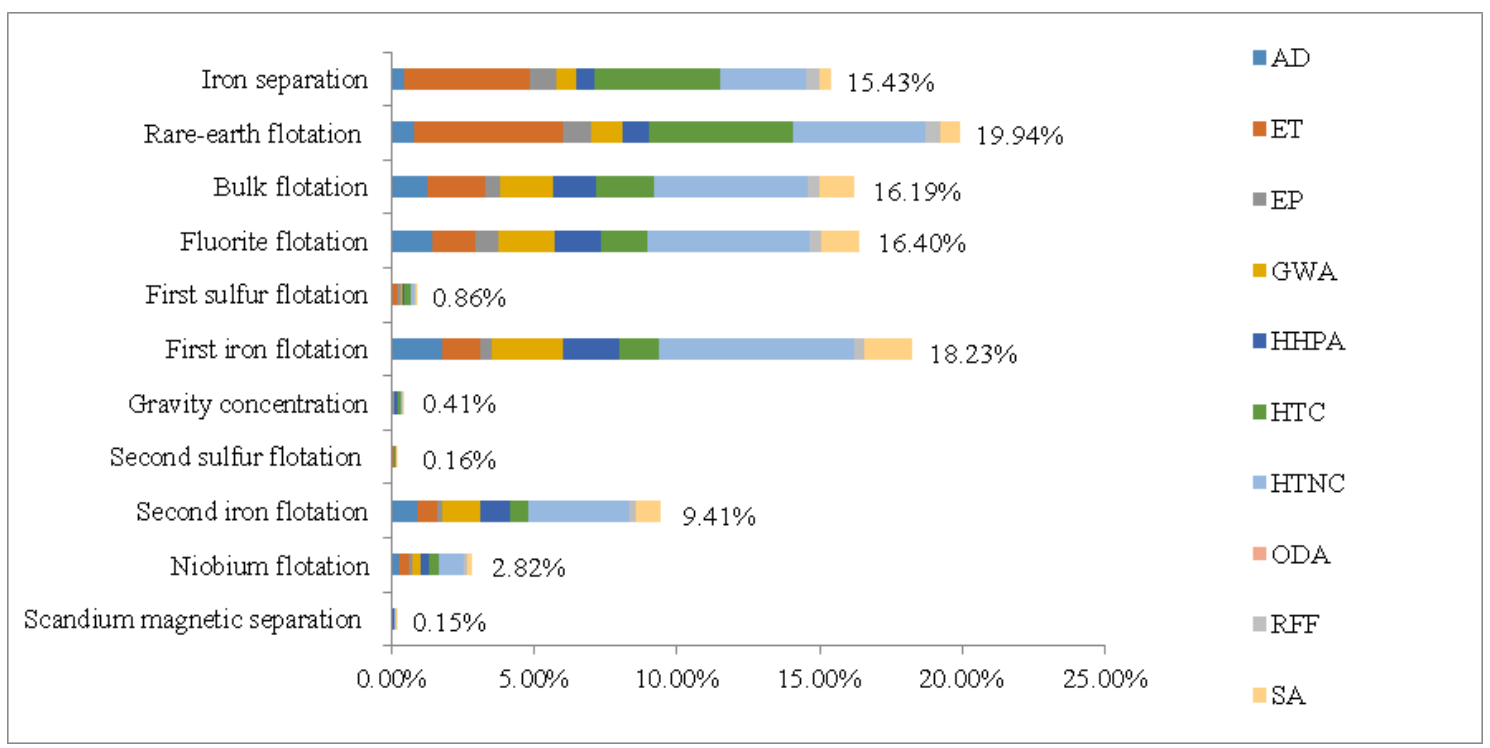

Figure 11. Proportion of each environmental impact in the total environmental impact

\section{Conclusions and suggestions}

\section{Conclusions}

(1) In LCA environmental impact types of integrated exploitation technology for tailings in Bayan Obo mine, HTNC is the main impact type and HTC is the next. All of these indicate that the major environmental problem in the processes of this technology is human health damage. The most serious process in HTNC is iron flotation. Steam is the major impact factor in HTNC, accounting for $96.33 \%$. The most serious process in HTC is rare-earth flotation. Water glass is the major impact factor in HTC, accounting for $83.16 \%$. From the above, it shows that environmental impact brought by raw material actually is much higher than that caused by pollution discharge in these actual processes. 
(2) In the total environmental impacts, flotation makes the heaviest pollution. Environmental pollution of some flotation processes is due to the production of flotation agents, such as rare-earth flotation. Environmental pollution of the other flotation processes is due to the pollution brought by energy production which meets the need of processes, such as iron flotation. Environmental impacts of rare-earth flotation and iron flotation account for $40 \%$, which is the largest proportion of the total environmental impacts.

\section{Suggestions}

(1) Based on this study, future studies about LCA of integrated exploitation technology for tailings in Bayan Obo mine may do some research into the different minerals such as rare-earth, iron, niobium, scandium et al. Meanwhile, the researchers should pay more attention to collecting or measuring the emission of wastewater and waste-gas in order to conduct a more accurate and objective assessment. Based on this study, the ecological design of products can be carried out, which could solve the problem of "end-of-pipe treatment" for comprehensively utilizing tailings.

(2) Because of the environmental impact brought by raw material is actually much higher than that caused by pollution discharge in the processes of tailings integrated exploitation of Bayan Obo mine, the rare-earth industry needs investigation and management in the selection and purchase of raw material. Therefore, green purchasing for flotation agent, inhibitor and collecting agent etc. is needed.

(3) If the producing technology and purchasing channel of raw material cannot be changed, the enterprises can conduct some contrast tests to find environmental friendly material for replacing previous material in technology. For the heaviest pollution of rare-earth flotation and iron flotation, technology itself should be deeply studies. The rare-earth industry not only keeps seeking green environmental substitute, but also needs to improve technology itself, reduce intermediate links as much as possible and improve the utilization rate of equipments.

Acknowledgements. This study was supported by Inner Mongolia University of Science and Technology Innovation Fund (grant number 2016QDL-B28); Inner Mongolia Autonomous Region Scientific Research Project of Higher Education Institution (grant number NJZY18152); Inner Mongolia Science \& Technology Plan (grant number 0901051701). We are thankful to Chen Shuilong from Gabi China for their kind help and guidance. We also thank Baotou Steel Group for their data support. Any opinions, findings, conclusions, or recommendations expressed in this study are those of the authors and do not necessarily reflect the views of Baotou Steel Group and related organizations.

\section{REFERENCES}

[1] Ahn, S. H., Chun, D. M., Chu, W. S. (2013): Perspective to green manufacturing and applications. - International Journal of Precision Engineering and Manufacturing 14(6): 873-874.

[2] Baotou Steel Group (2014): Yearbooks of Baotou Steel Group. - Inner Mongolia People's Publishing House, Hohhot.

[3] Baotou Steel Group (2016): Yearbooks of Baotou Steel Group. - Inner Mongolia People's Publishing House, Hohhot.

[4] Bonatz, N., Guo, R., Wu, W. H., Liu, L. J. (2019): A comparative study of the interlink ages between energy poverty and low carbon development in China and Germany by developing an energy poverty index. - Energy \& Buildings 183: 817-821. 
[5] Cao, H. C. (2017): Rare earth selection technology and flotation medicament study process. - Mineral Resources and Protection 3: 100-105.

[6] Che, L. P., Yu, Y. F. (2006): Present situation and development of rare earth mineral processing technology in China. - Rare Earth 27(1): 95-102.

[7] Chen, H., Shen, W. G., Shan, L. (2012): Discussion on iron tailings discharge and comprehensive utilization at home and abroad. - Concrete 2: 88-92.

[8] Cheng, J. Z., Hou, Y. B., Che, L. P. (2007): Reasonable exploitation and comprehensive utilization of rare earth resources in Bayan Obo deposit. - Rare Earth 28(1): 70-74.

[9] Hu, D., Clift, P. D., Böning, P., Hannigan, R., Hillier, S., Blusztajn, J., Wan, S., Fuller, D. Q. (2013): Holocene evolution in weathering and erosion patterns in the Pearl River delta. - Geochemistry Geophysics Geo Systems 14: 2349-2368.

[10] Hu, Q. B. (2014): Study on geological route selection for Baotou-Xi'an railway in largearea coal goaf region of Mu Us sandy land. - Railway Standard Design 3: 232-234.

[11] Ji, J. M. (2013): Recycle trail of iron and rare earth in Baotou steel flotation tailings. Metal Mine (3): 158-168.

[12] Jin, G., Li, Z. H., Lin, Q. W., Shi, C. C., Liu, B., Yao, L. N. (2015): Land Use Suitability Assessment in Low-Slope Hilly Regions under the Impact of Urbanization in Yunnan, China. - Advances In Meteorology, Article ID 848795.

[13] Jin, H. Y., Afiuny, P., McIntyre, T., Yih, Y., Sutherland, J. W. (2016): Comparative life cycle assessment of $\mathrm{NdFeB}$ magnets: virgin production versus magnet-to-magnet recycling. - 23rd CIRP Conference on Life Cycle Engineering 48: 45-50.

[14] Koltun, P., Tharumarajah, A. (2014): Life cycle impact of rare earth elements. - ISRN Metallurgy 4: 1-10.

[15] Li, C. L., Li, X. G., Xu, G. Y. (2015a): Comprehensive usage technology development and industrialization of concomitant mine in Bayan Obo. - Rare Earth 36(5): 151-158.

[16] Li, H. J., Li, Z. H., Li, Z. H., Yu, J., Liu, B. (2015b): Evaluation of ecosystem services: A case study in the middle reach of the Heihe River Basin, Northwest China. - Physics and Chemistry of The Earth 89-90: 40-45.

[17] Li, S., Zhai, L., Zou, B., Sang, H., Fang, X. (2017): A Generalized Additive Model Combining Principal Component Analysis for PM2.5 Concentration Estimation. - Isprs International Journal of Geo Information 6(8): 248.

[18] Li, Z. P., Fan, X. H., Yang, G. M. (2015c): Life cycle assessment of iron ore sintering process. - Journal of Iron and Steel Research 22: 473-477.

[19] Lima, F. M., Lovon-Canchumani, G. A., Sampaio, M., Tarazona-Alvarado, L. M. (2018): Life cycle assessment of the production of rare earth oxides from a Brazilian ore. Procedia CIRP 69: 481-486.

[20] Liobikienė, G., Grincevičienè, Š., Bernatonienė, J. (2017): Environmentally friendly behaviour and green purchase in Austria and Lithuania. - Journal of Cleaner Production 142: 3789-3797.

[21] Liu, D., Li, H., Wang, W., Zhou, C. (2015): Scenario forecast model of long term trends in rural labor transfer based on evolutionary games. - Journal of Evolutionary Economics 25: 649-670.

[22] Lu, H. H., Liu, H. M. (2016): Mine selection technology design of Baotou steel oxidative mine. - Modern Mine (11): 74-76.

[23] Mazzi, A., Toniolo, S., Catto, S. (2017): The combination of an environmental management system and life cycle assessment at the territorial level. - Environmental Impact Assessment Review 63: 59-71.

[24] Mei, G. J., Yu, J., Ge, Y. Y. (2006): Experimental study on the reduction of potassium and sodium content in Baotou iron concentrate by flotation. - Metal Mine (2): 31-35.

[25] Mo, H., Zhang, T. Z. (2003): Evaluation of data quality of life cycle list analysis. Environmental Science Research 16: 55-58.

[26] Navarro, J., Zhao, F. (2014): Life cycle assessment of the production of rare earth elements for energy applications: a review. - Frontiers in Energy Research 2: 1-17. 
[27] Ni, X., Cao, C., Zhou, Y., Cui, X., Singh, R. P. (2018): Spatio-Temporal Pattern Estimation of PM2.5 in Beijing-Tianjin-Hebei Region Based on MODIS AOD and Meteorological Data Using the Back Propagation Neural Network. - Atmosphere 9: 105.

[28] Norris, G. A. (2002): Impact characterization in the tool for the reduction and assessment of chemical and other environmental impacts: methods for acidification, eutrophication, and ozone formation. - Journal of Industrial Ecology 6: 79-101.

[29] Schulze, R., Lartigue-Peyrou, F., Ding, J., Schebek, L., Buchert, M. (2017): Developing a life cycle inventory for rare earth oxides from ion-adsorption deposits: key impacts and further research needs. - Journal of Sustainable Metallurgy 10: 1-19.

[30] Smith, M. P., Campbell, L. S., Kynicky, J. (2015): A review of the genesis of the world class Bayan Obo Fe-REE-Nb deposits, Inner Mongolia, China: multistage processes and outstanding questions. - Ore Geology Reviews (64): 459-476.

[31] Song, W., Deng, X., Liu, B., Li, Z., Jin, G. (2015): Impacts of Grain-for-Green and Grain-for-Blue Policies on Valued Ecosystem Services in Shandong Province, China. Advances In Meteorology, Article ID 213534.

[32] Sprecher, B., Xiao, Y. P., Walton, A., Speight, J., Harris, R., Kleijn, R., Visser, G., Kramer, G. J. (2014): Life Cycle inventory of the production of rare earths and the subsequent production of $\mathrm{NdFeB}$ rare earth permanent magnets. - Environmental Science and Technology 48: 3951-3958.

[33] Tian, J. D., Lu, Y. (1999): Research situation and production practice of rare earth recovery from iron tailings of Baotou steel concentrator. - Rare Earth 20(5): 54-58.

[34] Vahidi, E., Navarro, J., Zhao, F. (2016): An initial life cycle assessment of rare earth oxides production from ion-adsorption clays. - Resources, Conservation and Recycling 113: 1-11.

[35] Vahidi, E., Zhao, F. (2017): Environmental life cycle assessment on the separation of rare earth oxides through solvent extraction. - Journal of Environmental Management 203: 255-263.

[36] Vahidi, E., Zhao, F. (2018): Assessing the environmental footprint of the production of rare earth metal sand alloys via molten salt electrolysis. - Resources, Conservation \& Recycling 23: 178-187.

[37] Weng, Z. H., Haque, N., Mudd, G. M. (2016): Assessing the energy requirements and global warming potential of the production of rare earth elements. - Journal of Cleaner Production 139: 1282-1297.

[38] Weng, Z. H., Mudd, G. M. (2017): Global rare earth supply, life cycle assessment, and wind energy. - Wind Energy Engineering 34: 517-534.

[39] Xiao, R. G., Fei, H. C., An, G. Y., Zhang, H. C., Hou, W. R. (2003): Lithology and genesis of dolomite in Bayan'ebo mine, Inner Mongolia. - Geo Science 17: 287-293.

[40] Xie, J. Q., Zhang, J., Bai, Y. L. (2000): Analysis and discussion of mine selection technology process of baogang mine selection oxidative mine. - Mining and Metallurgical Engineering 9(3): 29-33.

[41] Xuan, W., Liu, J., Sun, C. (2009): Fundamental research on comprehensive exploitation and utilization of para genetic fluorite in Bayan Obo deposit of Inner Mongolia, China. Geo Science Front (s1): 178-178.

[42] Yan, H., Zhan, J., Liu, B., Huang, W., Li, Z. (2014): Spatially Explicit Assessment of Ecosystem Resilience: An Approach to Adapt to Climate Changes. - Advances In Meteorology, Article ID 798428.

[43] Yang, M. (2017): Item of 711 million of Baotou stell share purchasing comprehensive technology of strong stock holder. - Securities daily 5: 13-16.

[44] Yang, Y., Sun, L., Zhu, J., Wei, J., Su, Q., Sun, W., Liu, F., Shu, M. (2017): A simplified Suomi NPP VIIRS dust detection algorithm. - Journal of Atmospheric and Solar Terrestrial Physics 164: 314-323.

[45] Zaimes, G. G., Hubler, B. J., Wang, S. (2015): Environmental life cycle perspective on rare earth oxide production. - ACS Sustainable Chemistry and Engineering 2: 237-244. 
[46] Zapp, P., Marx, J., Schreiber, A., Friedrich, B., Voßenkaul, D. (2018): Comparison of dysprosium production from different resources by life cycle assessment. - Resources Conservation \& Recycling 130: 248-259.

[47] Zhai, L., Li, S., Zou, B., Sang, H., Fang, X., Xu, S. (2018): An improved geographically weighted regression model for PM2.5 concentration estimation in large areas. Atmospheric Environment 181: 145-154.

[48] Zhang, B., Xue, X. X., Yang, H., Huang, X. W. (2015): Gas-based magnetizing roasting for recycling iron in baotou rare earth tailings. - Journal of the Chinese Society of Rare Earths 3: 12-15.

[49] Zhang, W. H., Zheng, Y., Qin, Y. Q. (2002): Rare earth ore dressing from tailings of baotou steel concentrator. - Hydrometallurgy of China 21(1): 36-38.

[50] Zhang, Y., Ma, P. Q., Che, L. P. (2010): Experimental study on recovery of rare earth from Baotou steel tailings. - Chinese Rare Earths 31(2): 93-96.

[51] Zhao, X., Gao, Q., Sun, M., Xue, Y., Ma, R. Xiao, X., Ai, B. (2018): Statistical Analysis of Spatiotemporal Heterogeneity of the Distribution of Air Quality and Dominant Air Pollutants and the Effect Factors in Qingdao Urban Zones. - Atmosphere 9: 135.

[52] Zhou, Z. Q., Li, X. J., Chen, L. L., Li, B. Q., Liu, T. T., Ai, B. H., Yang, L. F., Liu, B., Chen, Q. (2018): Macrobenthic assemblage characteristics under stressed waters and ecological health assessment using AMBI and M-AMBI: a case study at the Xin'an River Estuary, Yantai, China. - Acta Oceanologica Sinica 37: 77-86. 